\title{
Cerebrospinal fluid outflow through skull channels instructs cranial hematopoiesis
}

Fadi E. Pulous ${ }^{1,2,3, \dagger}$, Jean C. Cruz-Hernández $1,3, \dagger$, Chongbo Yang ${ }^{1,2}$, Zeynep Kaya1,2, Gregory Wojtkiewicz ${ }^{1}$, Diane Capen 4 , Dennis Brown4, Juwell W. Wu1,3, Claudio Vinegoni1,2, Masahiro Yamazoe ${ }^{1,2}$, Jana Grune 1,2, Maximillian J. Schloss ${ }^{1,2}$, David Rohde ${ }^{1,2}$, Dmitry Richter ${ }^{1,3}$, Cameron S. McAlpine 5,6 , Peter Panizzi ${ }^{7}$, Ralph Weissleder 1,2 , Dong-Eog Kim ${ }^{8}$, Filip K. Swirski ${ }^{5}$, Charles P. Lin ${ }^{1,3^{*} \ddagger}$, Michael A. Moskowitz ${ }^{1,2,9^{*} \ddagger \text {, Matthias Nahrendorf } 1,2,10,11^{\star} \ddagger}$

${ }^{1}$ Center for Systems Biology, Massachusetts General Hospital and Harvard Medical School, Boston, MA, USA.

2Department of Radiology, Massachusetts General Hospital and Harvard Medical School, Boston, MA, USA.

3Wellman Center for Photomedicine, Massachusetts General Hospital and Harvard Medical School, Boston, MA, USA.

4Program in Membrane Biology, Division of Nephrology, Department of Medicine, Harvard Medical School and Massachusetts General Hospital, Boston, Massachusetts, USA.

${ }^{5}$ Cardiovascular Research Institute and Department of Medicine, Icahn School of Medicine at Mount Sinai, New York, NY, USA.

6Nash Family Department of Neuroscience, Icahn School of Medicine at Mount Sinai, New York, NY, USA.

7Department of Drug Discovery and Development, Harrison School of Pharmacy, Auburn University, Auburn, AL, USA.

8Molecular Imaging and Neurovascular Research Laboratory, Department of Neurology, Dongguk University College of Medicine, Goyang, South Korea.

9Department of Neurology, Massachusetts General Hospital and Harvard Medical School, Boston, MA, USA.

${ }^{10}$ Cardiovascular Research Center, Massachusetts General Hospital and Harvard Medical School, Boston, MA, USA.

11Department of Internal Medicine I, University Hospital Wuerzburg, Wuerzburg, Germany. tThese authors contributed equally.

¥These authors jointly supervised this work.

*Corresponding author. Email: charles_lin@hms.harvard.edu;

moskowitz@helix.mgh.harvard.edū; mnahrendorf@mgh.harvard.edu 


\begin{abstract}
Interactions between the immune and central nervous systems strongly influence brain health. Although the blood-brain barrier restricts this crosstalk, we now know that meningeal gateways through brain border tissues, particularly dural lymphatic vessels that allow cerebrospinal fluid outflow, facilitate intersystem communication. Here we observe that cerebrospinal fluid exits into the skull bone marrow. Fluorescent tracers injected into the cisterna magna of mice travel through hundreds of sub-millimeter skull channels into the calvarial marrow. During meningitis, bacteria usurp this perivascular route to infect the skull's hematopoietic niches and initiate cranial hematopoiesis ahead of remote tibial sites. Because skull channels also directly provide leukocytes to meninges, the privileged sampling of brain-derived danger signals in cerebrospinal fluid by regional marrow has broad implications for neurological disorders.
\end{abstract}

\title{
One-Sentence Summary
}

Skull channels transport cerebrospinal fluid from the subarachnoid space to the cranial bone marrow via a perivascular route, which bacteria use during meningitis. 


\section{Introduction}

In addition to guarding brain health, the immune system participates in a wide array of neurological disorders. The blood-brain barrier enforces an unusually rigid leukocyte origin dichotomy, dividing central nervous system (CNS)-resident immune cells from systemically circulating leukocytes. Crosstalk between local brain and systemic immune system components is limited in the steady state but expands during pathologies. Residing at potential portals of entry, the meninges are CNS border tissues that provide the brain and spinal cord with a protective connective tissue capsule consisting of three tissue layers (pia mater, arachnoid and dura mater) within which cerebrospinal fluid (CSF) flows between the arachnoidal membrane and pia mater. A complementary glymphatic system (1), which drains the brain's interstitial and perivascular spaces, interfaces with CSF. Ultimately, CSF exits into the venous blood and the lymphatics $(2,3)$.

The CNS border tissues dynamically police leukocyte migration and brain-derived signal exit. Recently discovered skull channels connecting the cranial bone marrow to the meninges, in mice and humans, constitute a novel leukocyte portal into the CNS (4-6). Skull hematopoietic activity directly adjacent to the brain delivers myeloid cells $(4,6)$ and B lymphocytes $(5)$, bypassing the blood-brain barrier. If the skull marrow is indeed a private leukocyte purveyor for the brain, skull-derived immune cells may be an intermediate third entity between resident and non-resident CNS leukocytes. Further, dural lymphatics provide a CSF outflow which freely exchanges with the brain's glymphatic system (1). This signaling pathway shares information about brain health systemically, allowing for presentation of CNS-derived antigen in cervical lymph nodes and consequently activating adaptive immunity $(7,8)$. Collectively, these reports expand our understanding of immune cell function across CNS borders and specifically implicate CSF as an under-appreciated messenger that may coordinate neuroinflammation.

Here we describe previously unrecognized CSF outflow into the skull bone marrow. Fluorescent tracers injected into the cisterna magna of mice migrate along the perivascular spaces of dural 
blood vessels and then perivascularly travel through skull channels into the cranial marrow. In mice with meningitis, bacteria usurp this path into the skull marrow, thereby boosting cranial emergency hematopoiesis. 


\section{Results}

\section{Perivascular CSF transit through skull channels into marrow cavities}

To understand the spatial organization of the skull channels, we first performed high-resolution ex vivo X-ray computed tomography of the skull (Fig. 1A) and characterized regional channel networks overlying frontal, parietal and occipital brain lobes (Fig. 1B). Channels traversed the inner compact bone into the marrow-containing cavities. We observed the highest density of skull channels in the frontal and occipital regions (Fig. 1C). Given a CT-derived mean channel density above 10 per $\mathrm{mm}^{2}$ and an inner skull surface area $>100 \mathrm{~mm}^{2}$, we estimate that more than 1,000 channels reach into the cranial vault of an adult mouse. Frontal and parietal skull channels formed the shortest connections to the dura, ranging from $83-90 \mu \mathrm{m}$, whereas occipital skull channels were approximately $25 \%$ longer (Fig. 1D). Frontal and parietal skull channels were $20 \%$ narrower than their occipital counterparts (Fig. 1E), pointing to regional channel heterogeneity.

We hypothesized that these numerous links between the skull marrow and the dura enable not only cell traffic towards the meninges (4) but also bidirectional crosstalk. We therefore implemented a 2-photon intravital microscopy (IVM) and ex vivo imaging pipeline to visualize CSF distribution after fluorescent tracer injection into the cisterna magna $(9,10)$. Intracisternal injection of $70 \mathrm{kD}$ FITC-labeled ovalbumin was combined with intravenous labeling of the blood pool using $70 \mathrm{kD}$ Texas red-labeled dextran. We observed a striking perivascular appearance of the intracisternal tracer (Movie S1 and Fig. 1F) along a subset of dural vessels, indicating that CSF travels perivascularly from the subarachnoid space into the dura. This led us to wonder whether we could track this CSF outflow into the skull marrow cavity. We labeled cranial vasculature with intravenously injected fluorescently conjugated CD31/Sca1 antibodies and CSF with intracisternally injected fluorescently labeled ovalbumin, followed by intravital imaging of the skull marrow cavity $(4,11,12)$. In the skull marrow, we detected perivascular spaces and cells labeled with the intracisternally injected CSF tracer (Fig. 1G). This critical observation in 
the dura and marrow suggested that perivascular CSF outflow into the marrow may occur through skull channels.

As we anticipated, high magnification transmission electron microscopy analysis of skull channels revealed a perivascular space that may accommodate CSF transport beginning at the dural channel opening (Fig. 1H). To directly test this hypothesis, we labeled cranial vasculature with intravenous Texas red dextran and the CSF with an intracisternal FITC dextran injection one hour prior to ex vivo microscopy of the inner skull cortex. Channel cross sections were clearly demarcated by dextran-labeled vessels surrounded by bone visualized with second harmonic generation (Fig. 1I). Z-stacks that began on the dural surface and moved deep into the marrow cavity (Fig. 1I and Movie S2) visualized intracisternally injected dextran in numerous skull channels. This signal surrounded the blood vessel and was present from channels' dural openings all the way into the marrow cavity. Counting CSF-tracer-containing channels revealed that $67 \%$ of them showed perivascular signal after intracisternal dextran injection (Fig. 1J). Bone marrow imaging after intracisternal injection revealed a similar perivascular appearance for tracers with molecular weights from $66 \mathrm{kD}$ to $2000 \mathrm{kD}$ along a subset of skull marrow vessels (Fig. 1K). Labeled CSF was detected in the skull marrow as early as 15-30 minutes after injection but was largely absent from the tibia's marrow vasculature (Fig. 1G and S1); this indicates that CSF was excluded from systemic circulation at early time points. Together, these data demonstrate that CSF exits the subarachnoid space via perivascular flux along a subset of dural vessels which connect into the bone marrow cavity through an extensive skull channel network. This finding implicates the skull marrow as a CSF-sensing hematopoietic compartment. We next sought to examine these observations' relevance in a mouse model of bacterial meningitis.

\section{Streptococcus pneumoniae expand near dural skull channel openings}

We adapted a model of pneumococcal meningitis (13) to test the functional significance of skull channel connections in neuroinflammation. Streptococcus pneumoniae is the clinically dominant 
cause of bacterial meningitis (14). To establish a disease timeline in mice, we injected $5 \times 10^{3}$ bioluminescent Streptococcus pneumoniae Xen10 bacteria into the cisterna magna and analyzed bacterial propagation over time alongside control mice that received an equal volume of artificial CSF (Fig. 2A). Whole-body bioluminescence (BLI) imaging revealed a timedependent signal increase reporting bacterial growth (Fig. 2B). BLI signal was observed predominantly in the skull 36 hours after injection and by 48 hours had spread to the spine. Bacterial burden increased exponentially by 36 hours, with a peak at 48 hours after injection (Fig. 2C). To assess meningeal inflammation, we measured the canonical inflammatory cytokines $/ / 1 \beta, / / 6$ and TNF $\alpha$ in the meninges by qPCR and found them to be 40 - to 60 -fold higher in mice with meningitis relative to controls injected with artificial CSF (Fig. S2). We next assessed S. pneumoniae growth in the blood and CSF using a bacteria colony forming unit assay 48 hours after infection. The blood contained a miniscule amount of $S$. pneumoniae, while the CSF contained approximately 10,000-fold more. This suggests that 48 hours after infection, bacterial propagation is mostly confined to the meninges (Fig. 2D). We next sought to visualize S. pneumoniae manifestation relative to the skull marrow cavity and skull-dural channel connections. To this end, we injected green fluorescent protein (GFP)-expressing $S$. pneumoniae strain D39V hlpA-GFP into the cisterna magna and adapted an optical clearing protocol (15) to show skull channels, the marrow cavity and adjacent bacterial propagation in the subarachnoidal space. The skull marrow vasculature was stained with intravenously injected fluorescent CD31/Sca-1 antibodies and the bone with osteosense, allowing us to identify intact marrow, channels and the CSF space by confocal microscopy (Fig. 2E). To visualize GFP+ S. pneumoniae, we employed the timeline established by bioluminescence analysis (Fig. 2A-C). We injected $5 \times 10^{3} \mathrm{GFP}+$ S. pneumoniae, or an equal volume of artificial CSF in controls, and sacrificed mice 48 hours later (Fig. 2F). Confocal microscopy of cleared tissue revealed abundant GFP+ bacterial growth in the subarachnoid space of mice with S. pneumoniae meningitis, but not controls (Fig. 2E, G and Movie S3). Three-dimensional reconstructions document the proximity of GFP+ S. pneumoniae to skull channels (Fig. $2 \mathrm{H}$ and Movie S4). In addition to subarachnoidal bacterial colonies directly adjacent to dural skull channel openings, a 
smaller number of bacteria were also present in the skull marrow's extravascular space (Fig. $2 \mathrm{H})$. Taken together, these findings gave rise to the hypothesis that in meningitis, bacteria may enter the skull bone marrow. While we know skull fractures may cause bacterial meningitis, intact skull invasion from within the cranial vault has not been explored previously.

\section{Pneumococcal meningitis propagates to the skull}

To test the hypothesis that S. pneumoniae enter the skull marrow cavity, we performed in vivo confocal microscopy of the intact skull in mice with meningitis. Mice were imaged 48 hours after intracisternal injection with GFP+ S. pneumoniae or artificial CSF in controls. The vasculature was labeled with intravenous CD31/Sca-1 antibodies one hour prior to imaging and the bone was labeled via osteosense injection 24 hours prior to imaging. Surprisingly, we observed GFP+ bacterial colonies in the skull marrow extravascular spaces of mice with meningitis (Fig. 3A, Movie S6). No such signal was detectable in controls (Fig. 3A and Movie S5). Since this, to our knowledge, is the first observation of $S$. pneumoniae entering the skull cavity during meningitis, we sought to corroborate these imaging data with orthogonal assays, including bacterial cultures, qPCR for bacterial genes and flow cytometry to detect GFP expressed by bacteria.

Using an experimental timeline (Fig. 3B) comparable to the microscopy experiments described above, we first employed a bacterial colony forming unit (CFU) assay to analyze bacterial growth 48 hours after intracisternal injection of S. pneumoniae. Tibia and skull bone marrow was harvested, homogenized and plated on blood agar plates to accommodate bacterial colony growth, similar to clinical blood cultures. While bacteria were nearly undetectable in tibial marrow, bacterial colonies grew from the skull marrow preparations (Fig. 3C, D). Since only viable bacteria can divide, this finding documents that live bacteria were present in the skulls of mice with meningitis. We next compared skull and tibial marrow using qPCR analysis for the bacterial gene $p s a A$, which is not expressed in mice. Skull samples from mice with meningitis contained markedly higher levels of psaA transcript compared to the tibia (Fig. 3E), confirming the presence of $S$. pneumoniae within the skull marrow. Furthermore, we performed flow 
cytometric analysis on skull marrow isolated from mice after intracisternal injection of GFP+ $S$. pneumoniae. In addition to detecting bacterial presence, flow cytometry also determined whether bacteria are located inside cells. The skulls of mice with meningitis showed substantial numbers of CD45+ GFP+ leukocytes, which were largely absent in the skulls of control mice (Fig. 3F). We document the observed fraction of bacteria-containing leukocytes in a right-shifted GFP histogram in CD45+ leukocytes obtained from the skull marrow of mice with meningitis, as compared to controls injected with artificial CSF (Fig. 3G and 3H). We also analyzed the cellfree supernatant of bone marrow suspensions following high-speed centrifugation by flow cytometry. In the skull marrow supernatant obtained from mice that received intracisternal GFP+ S. pneumoniae injection, we noted abundant bacteria that were absent in controls (Fig. 3I). Taken together, the imaging observation of bacteria in the skull marrow of mice with meningitis was confirmed by three independent assays, all supporting that bacteria can propagate from the meninges to the skull marrow.

\section{Skull channels are conduits for S. pneumoniae to access the calvarial marrow}

We next sought to directly evaluate whether $S$. pneumoniae reach the skull marrow by transiting skull channels from the dura. Accordingly, we performed ex vivo confocal microscopy of tissuecleared skull preparations containing intact brain tissue 48 hours after intracisternal injection of either GFP+ S. pneumoniae or artificial CSF in controls. Z-stack projections of skull channels revealed the presence of GFP+ S. pneumoniae both in the dura and inside skull channels (Fig. 4A). We found that $75 \%$ of mice with meningitis showed bacterial GFP signal in their skull channels, whereas control animals without meningitis lacked any sign of S. pneumoniae (Fig. 4B). Three-dimensional reconstructions of skull channels revealed extravascular GFP localization within the channels of mice with meningitis, but not in controls (Fig. 4C and Movies S7 and S8). As a complementary approach to imaging bacterial GFP directly, we adapted the CUBIC method to search for GFP+ S. pneumoniae in deeper tissue areas (15). More specifically, we modified the CUBIC protocol II (15) to include delipidation, decolorization, decalcification, refractive-index matching and ultimately immunostaining for bacterial GFP with a 
fluorescently labeled anti-GFP antibody. Whole-mount confocal microscopy of CUBICprocessed specimens revealed a striking pattern of anti-GFP staining within skull channels and in the dura mater (Fig. 4D). Skulls from control mice showed no GFP signal in the dura, channels or marrow (Fig. 4D). Collectively, these data demonstrate two critical and previously unknown phenomena: i) during meningitis, bacteria enter the skull marrow through skull channels from the dura, and ii) bacterial influx through channels into the marrow likely occurs via a perivascular route, similar to CSF outflow into the marrow as observed in the steady state.

\section{S. pneumoniae propagation within the CSF induces a skull-specific increase in LSK}

\section{proliferation}

Having defined the route by which S. pneumoniae transit skull channels via perivascular passage and arrive within the skull marrow, we next tested how this bacterial expansion functionally affects hematopoiesis. Specifically, we devised an experimental timeline to test whether intracisternal injection of $S$. pneumoniae elicits a hematopoietic response in skull marrow before distal tibial bone marrow (Fig. 5A). We hypothesized that skull marrow changes may be observable as early as 6 hours following intracisternal injection of $1 \times 10^{5} \mathrm{~S}$.

pneumoniae. Our prior work, which supports this timeline, had identified a rapid skull marrow response in an ischemic stroke model (4). We first performed qPCR to clarify if bacteria enter the skull at this early time point and found that the bacterial gene $p s a A$ was indeed expressed in the skull but not the tibia (Fig. 5B) 6 hours after intracisternal infection. We then analyzed BrdU incorporation into Lin- Sca1+ ${ }^{+}$-kit ${ }^{+}$hematopoietic progenitors (LSK) in the skull and tibia by flow cytometry to determine whether S. pneumoniae had altered LSK proliferation. Mice injected with bacteria had significantly increased BrdU+ LSK in their skulls but not in their tibial marrow (Fig. 5C, D). To confirm our observation that elevated LSK proliferation associates with direct local S. pneumoniae skull infiltration, we used confocal IVM to image GFP+ S. pneumoniae progression into the skull marrow 3 hours after injecting bacteria into the cisterna magna. To explore if bacteria were located in the skull marrow's CSF-containing compartment, we co-injected fluorescently labeled ovalbumin at the time of bacterial infection (Fig. 5E). We noted large 
extravascular GFP+ signal clusters, within the marrow, that were co-labeled with intracisternally injected ovalbumin (Fig. 5F, 5F'), a result suggesting that cells contained bacteria. Additionally, we observed smaller extravascular $\mathrm{GFP}^{+}$areas that also colocalized with the CSF tracer ovalbumin, and we interpreted these as extracellular bacteria (Fig. 5F"). In sum (Fig. 5G), these data point to a process by which intracisternally injected bacteria co-opt a perivascular CSF passage into the skull marrow, inciting a skull-specific increase in LSK proliferation that precedes changes in distal tibial bone marrow. 


\section{Discussion}

Every day, the human ventricular choroid plexus produces most of the $500 \mathrm{ml} \mathrm{CSF}$ that provides a protective environment for the brain and receives waste from brain interstitial fluid through exchange with the glymphatic system. CSF outflow is thought to occur via several routes (2): i) through arachnoidal villi described more than one hundred years ago (16), ii) along spinal and cranial nerves and iii) through dural lymphatics to cervical lymph nodes $(17,18)$. Our work establishes a previously unrecognized outward signaling pathway facilitated by CSF exit along dural vessels that transit skull channels into the marrow.

Given that skull channel CSF outflow - like dural lymphatic vessels - connects to a site of leukocyte abundance, it likely also serves immune surveillance. We speculate that while dural lymphatics alert adaptive immune cells residing in lymph nodes, skull channel signaling may primarily involve innate immune cells produced locally in the calvarial marrow. This reasoning is supported by reports of innate immune cell migration through skull channels in steady state, nerve crush injury, experimental autoimmune encephalomyelitis (6) (a mouse model of multiple sclerosis) and chemically induced meningitis (4). In contrast to meningeal lymphatics, skull channels transport CSF in a perivascular fashion and facilitate bidirectional exchange. Moreover, skull channels may target spatially distinct areas within meninges, brain and spinal cord because there are many channels that distribute widely within the skull and vertebrae. When considered together, CSF outflow to skull marrow and leukocyte migration towards the meninges appear to be components of a local immune cell supply chain that bypasses systemic circulation, another important distinction from meningeal lymphatics.

During meningitis, the skull marrow is a prominent provider of neutrophils, which reach the meninges through skull channels (4). Neutrophils are early responders that combat bacteria in the meninges but also inflict irreversible nerve damage, a common long-term problem in meningitis survivors (19). Better understanding skull hematopoiesis as well as neutrophil supply, function and subsets may provide opportunities to support bacterial clearance while alleviating 
inflammatory CNS damage. The presence of live extracellular bacteria in the skull marrow suggests this hematopoietic tissue may indeed be infected. Intracellular bacteria, on the other hand, may have been removed from the CNS by phagocytes, cells capable of antigen presentation. Bacterial meningitis is most prevalent in newborns and the elderly (19), age ranges associated with decreased skull marrow function. The skull is not yet fully developed in neonates, and skull marrow undergoes fatty replacement in advanced age $(20,21)$. We therefore speculate that the bacterial meningitis incidence peaks in those age groups could be related to not only the immune system's changing competency but also lower hematopoietic capability of the skull marrow. This notion is supported by our observation that hematopoiesis expansion in the cranium outpaced the remote tibial response. Important open questions include whether the phenotype of skull-derived neutrophils differs from cells made in distant marrow regions and how exactly the skull marrow is activated. Prior data on infection-induced emergency hematopoiesis indicate that Toll-like receptors likely sense bacteria (22), either directly by hematopoietic progenitors or indirectly via hematopoietic niche cells (Immgen database and deposited data from ref (23)).

Our work introduces CSF sampling by the skull marrow, in which immune sentinels are highly abundant. Perhaps related to this discovery, recent human imaging studies showed robust inflammatory signal in skull bone marrow overlying repeatedly abnormal brain cortex in patients with migraine (24). While we demonstrate the relevance of CSF outflow to the skull in the setting of bacterial meningitis in mice, such surveillance likely also shapes the immune response in chronic inflammatory CNS disorders such as Alzheimer's disease and multiple sclerosis. It remains to be determined which cells, cytokines, danger signals, antigens and small molecules traffic through skull channels and how such signaling regulates the production, phenotypes and migration of skull-derived leukocytes towards the CNS. Since dura - marrow connections similar to skull channels exist in vertebrae (25), the question arises if vertebral marrow reacts in synchrony with the skull or more like the remote tibial marrow. Generally, the skull marrow warrants closer scrutiny due to its proximity to and crosstalk with the meninges and the CNS. 
Constant sampling of CSF outflow suggests the skull marrow state may reflect brain health and that the skull marrow has a prominent role in regulating CNS inflammation.

\section{References and Notes}

1. J. J. Iliff et al., Sci Transl Med 4, 147ra111 (2012).

2. S. T. Proulx, Cell Mol Life Sci 78, 2429 (2021).

3. B. A. Plog, M. Nedergaard, Annu Rev Pathol 13, 379 (2018).

4. F. Herisson et al., Nat Neurosci 21, 1209 (2018).

5. S. Brioschi et al., Science (2021).

6. A. Cugurra et al., Science (2021).

7. C. Harling-Berg, P. M. Knopf, J. Merriam, H. F. Cserr, J Neuroimmunol 25, 185 (1989).

8. M. G. Harris et al., Sci Rep 4, 4422 (2014).

9. E. H. Stanton et al., Magn Reson Med 85, 3326 (2021).

10. A. L. R. Xavier et al., J Vis Exp (2018).

11. G. Courties et al., Circ Res 116, 407 (2015).

12. K. Vandoorne et al., Circ Res 123, 415 (2018).

13. B. Mook-Kanamori, M. Geldhoff, D. Troost, T. van der Poll, D. van de Beek, BMC Infect Dis 12, 71 (2012).

14. D. van de Beek, J. de Gans, A. R. Tunkel, E. F. Wijdicks, N Engl J Med 354, 44 (2006).

15. K. Tainaka et al., Cell Rep 24, 2196 (2018).

16. L. H. Weed, J Med Res 31, 51 (1914).

17. A. Louveau et al., Nature 523, 337 (2015).

18. A. Aspelund et al., J Exp Med 212, 991 (2015).

19. B. B. Mook-Kanamori, M. Geldhoff, T. van der Poll, D. van de Beek, Clin Microbiol Rev 24, 557 (2011).

20. T. H. Ambrosi et al., Cell Stem Cell 20, 771 (2017).

21. S. W. Jin, K. B. Sim, S. D. Kim, J Korean Neurosurg Soc 59, 192 (2016).

22. N. A. Lind, V. E. Rael, K. Pestal, B. Liu, G. M. Barton, Nat Rev Immunol (2021).

23. A. N. Tikhonova et al., Nature 569, 222 (2019).

24. N. Hadjikhani et al., Ann Neurol 87, 939 (2020).

25. H. Yao et al., Nature 560, 55 (2018).

26. M. Kjos et al., J Bacteriol 197, 807 (2015).

27. G. Carvalho Mda et al., J Clin Microbiol 45, 2460 (2007).

\section{Acknowledgements}

We acknowledge Jan-Willem Veening for providing fluorescent bacteria and Kaley Joyes for editing the manuscript.

\section{Funding}

This work was funded in part by U.S. federal funds from the National Institutes of Health (HL158040, HL142494, HL139598, HL125428, NS108419 and HL135752) and the Korean National Research Foundation (NRF-2020R1A2C3008295 and NRF-2021R1A6A1A03038865).

\section{Author contributions}

F.E.P. conceived the study; designed, performed and analyzed imaging and wet lab experiments; induced meningitis; interpreted data and made the figures. J.C.C.-H. designed, performed and analyzed imaging experiments. C.Y. and Z.K. optimized the meningitis model for imaging assays; performed and analyzed experiments; interpreted data and discussed strategy. G.W., D.C., M.Y, J.G., M.J.S. and D. Ro. performed experiments and collected data. C.V. participated in optical clearing and imaging experiments. D.Ri. and J.W.W. provided image analysis. D.B., C.V., D.E.K., F.K.S. and R.W. discussed data and experimental design. F.E.P. 
bioRxiv preprint doi: https://doi.org/10.1101/2021.08.27.457954; this version posted August 28, 2021. The copyright holder for this preprint (which was not certified by peer review) is the author/funder. All rights reserved. No reuse allowed without permission.

and M.N. wrote the manuscript with input from all authors. C.L., M.A.M. and M.N. conceived and directed the study.

\section{Competing interests}

The authors declare no competing interests. 
Figures and legends

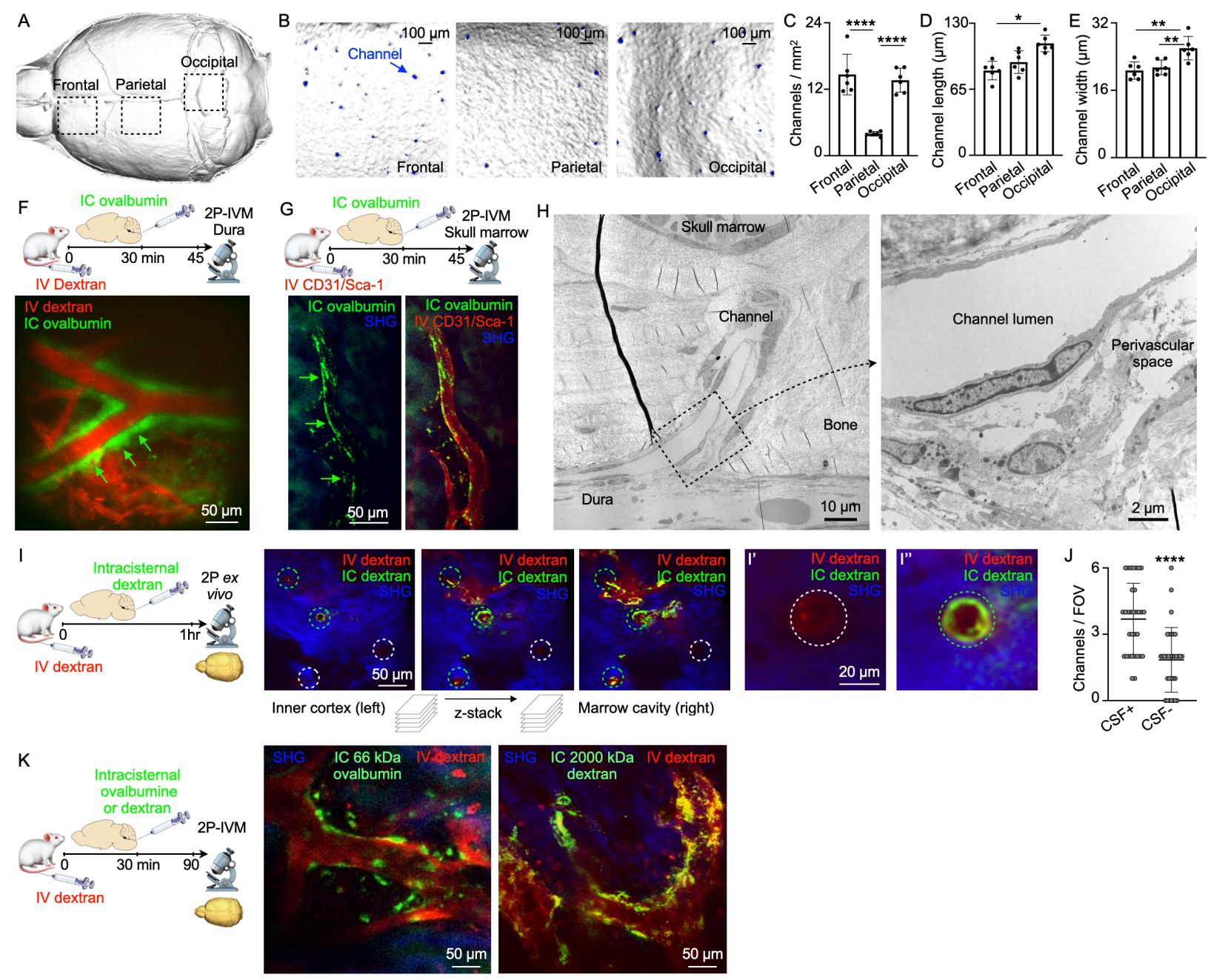

Fig. 1. A perivascular space in skull channels facilitates cerebrospinal fluid outflow into the cranial marrow. (A) Inner skull cortex 3D surface reconstruction from microCT. (B) 3D reconstructions of inner frontal, parietal and occipital bone surfaces with channel openings indicated in blue(scale bar: $100 \mu \mathrm{m})$. Regional channel density (C), length (D) and width (E) measured by microCT (mean $\pm \mathrm{SD}$; $\mathrm{n}=6$ mice; one-way ANOVA with Tukey's multiple comparisons test; $\left.{ }^{*} P<.05,{ }^{* *} P<.01,{ }^{* * *} P<.0001\right)$. (F) Scheme and intravital microscopy (IVM) image of intracisternally (IC) injected ovalbumin in the perivascular space of a dural vessel intravenously (IV) labeled with dextran (scale: $50 \mu \mathrm{m}$ ). (G) Scheme and IVM image of intracisternally injected ovalbumin in the perivascular space of a bone marrow vessel (scale: 50 $\mu \mathrm{m})$. (H) Transmission electron microscopy of a skull channel in the frontal bone (scale: $10 \mu \mathrm{m})$. Inset of perivascular area (scale: $2 \mu \mathrm{m}$ ). (I) Z-stack of interior skull cortex ex vivo microscopy in mice after intracisternal and intravenous injections of dextran. Bone signal from second harmonic generation surrounds channels (circles). I' and I" are insets of IC tracer negative and positive channels (scale: $50 \mu \mathrm{m}$ and $20 \mu \mathrm{m}$ ). (J) Number of CSF-containing channels (mean \pm SD; $n=3$ mice; Mann-Whitney test; $P<.0001$ ). (K) IVM of mice after intracisternal injection of ovalbumin/dextran for CSF tracing and IV dextran (scale: $50 \mu \mathrm{m}, \mathrm{n}=3-5$ mice per tracer; scale: $50 \mu \mathrm{m})$. 
A

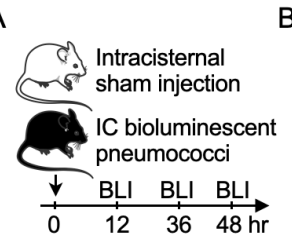

$\mathrm{E}$
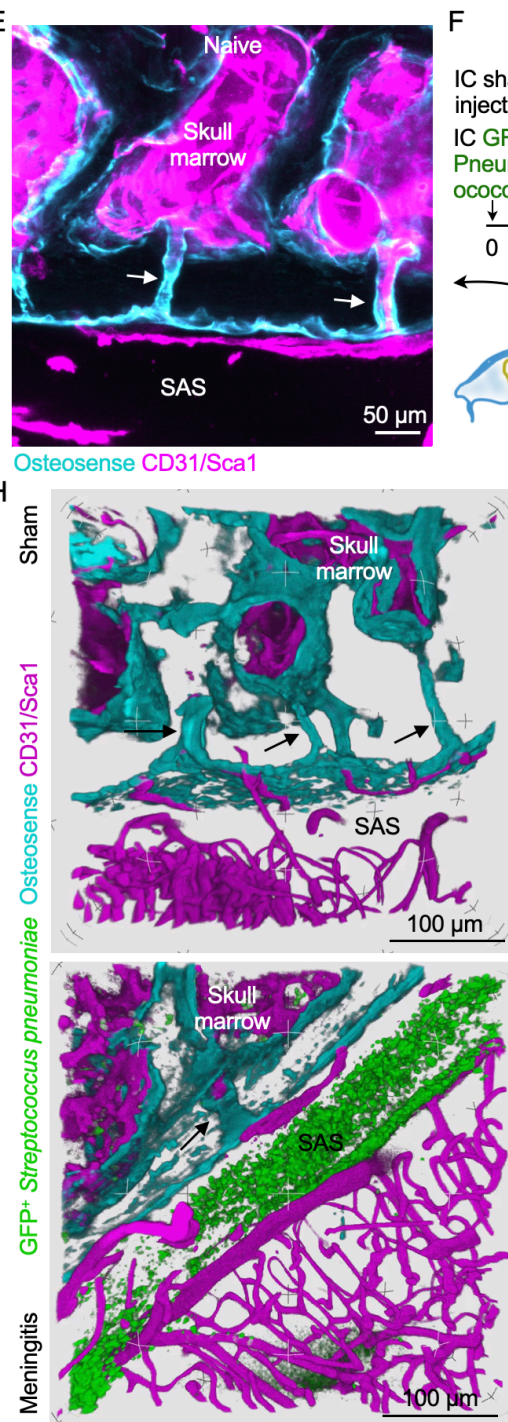
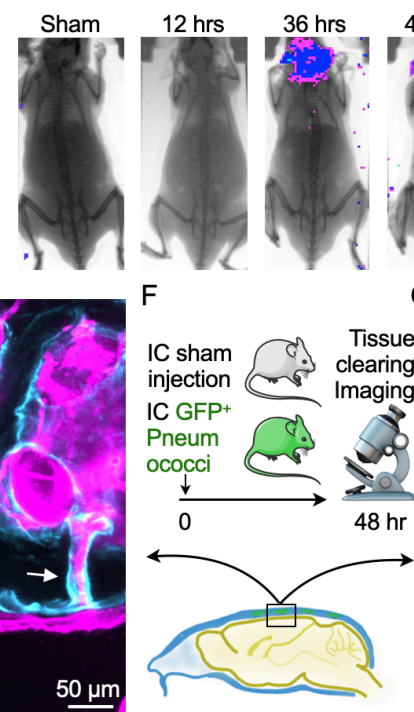
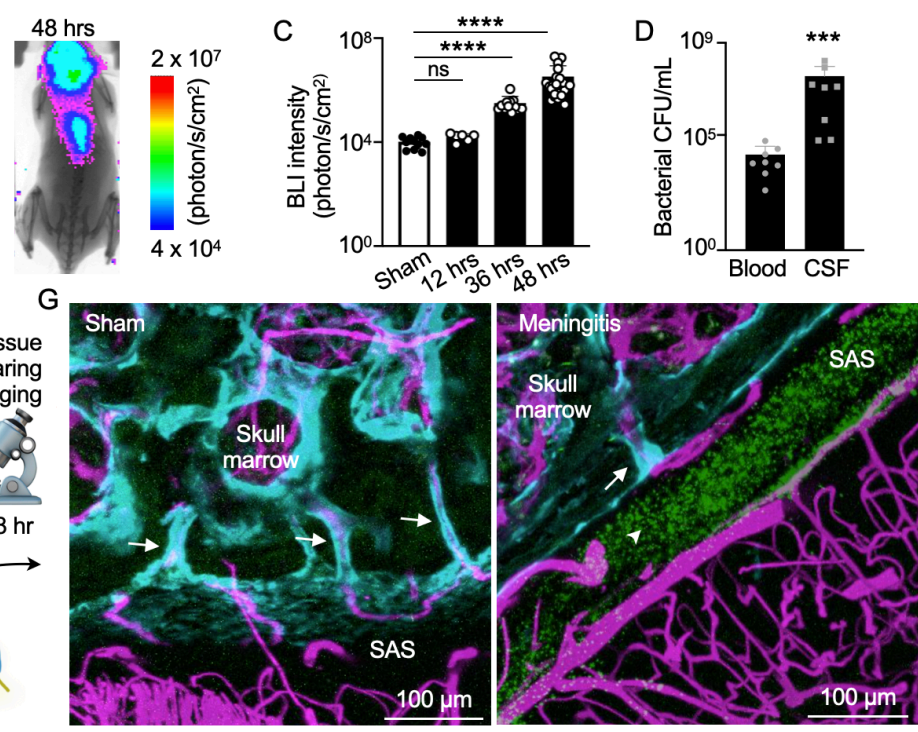

Osteosense CD31/Sca1 GFP+ Streptococcus pneumoniae
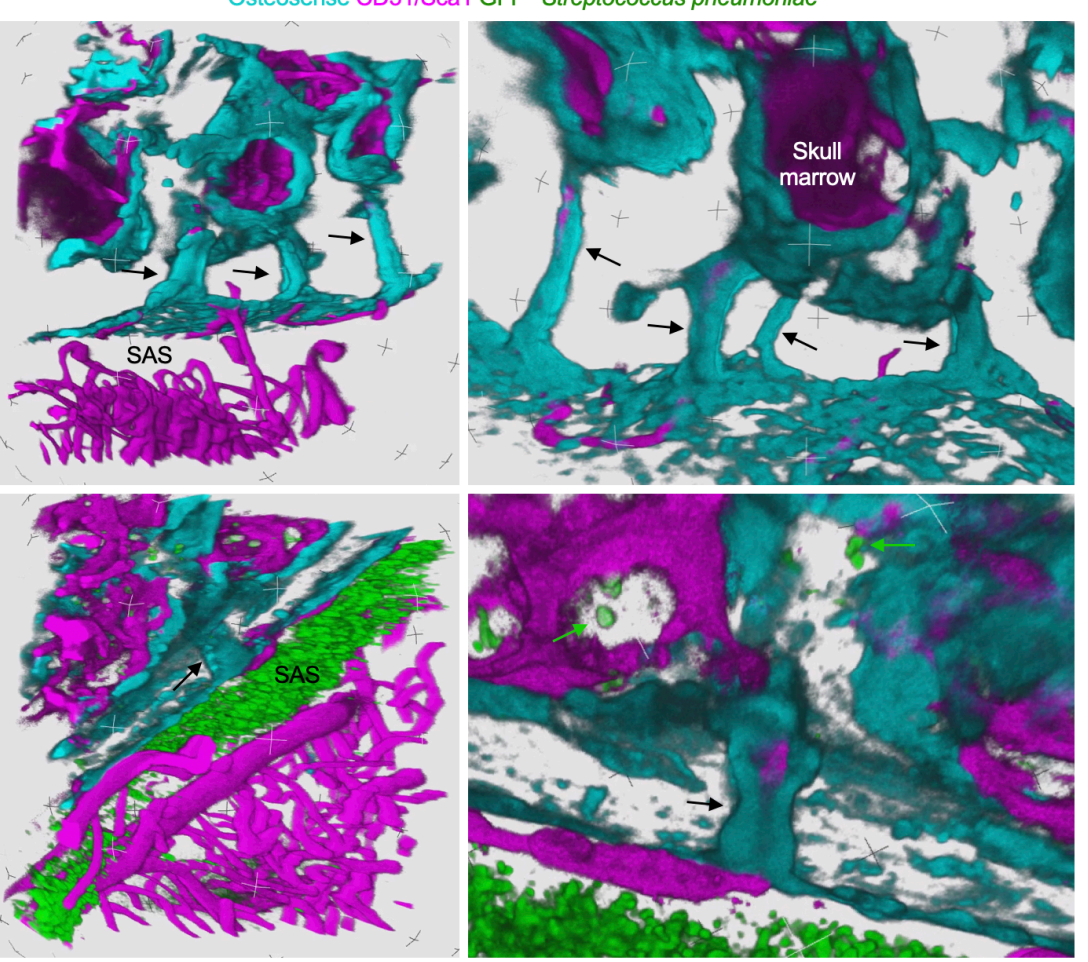

Fig. 2. Bacterial propagation during pneumococcal meningitis. (A) Timeline for bioluminescent Streptococcus pneumoniae Xen10 meningitis. (B) Bioluminescence imaging (BLI) of sham controls or mice after intracisternal injection of $S$. pneumoniae Xen10 at indicated timepoints. (C) Quantitation of bacterial load with BLI (mean \pm SD; $n=6-24$ mice; Kruskal-Wallis test with Dunn's multiple comparisons test; ns=not significant, ${ }^{*} P<.05$, $\left.{ }^{* * * *} P<.0001\right)$. (D) Bacterial colony forming unit (CFU) assay from blood and CSF $48 \mathrm{hrs}$ after infection (mean \pm SD; $\mathrm{n}=8$ mice; Mann-Whitney Test; ${ }^{* * *} P<.001$ ). (E) Skull channels visualized after tissueclearing (scale: $50 \mu \mathrm{m}$ ). (F) Experimental outline for tissue clearing and meningitis imaging. (G) Representative images of sham controls or mice after intracisternal injection of Streptococcus pneumoniae JWV500. Osteosense identified bone while IV CD31-Sca1 labeled BM and dural vessels ( $n=4-6$ mice; scale: $100 \mu \mathrm{m})$. $(\mathbf{H}) 3 \mathrm{D}$ reconstructions of $(G)$ highlighting skull channels (arrows) and bacterial propagation in marrow (scale: $100 \mu \mathrm{m}$ ). 
A
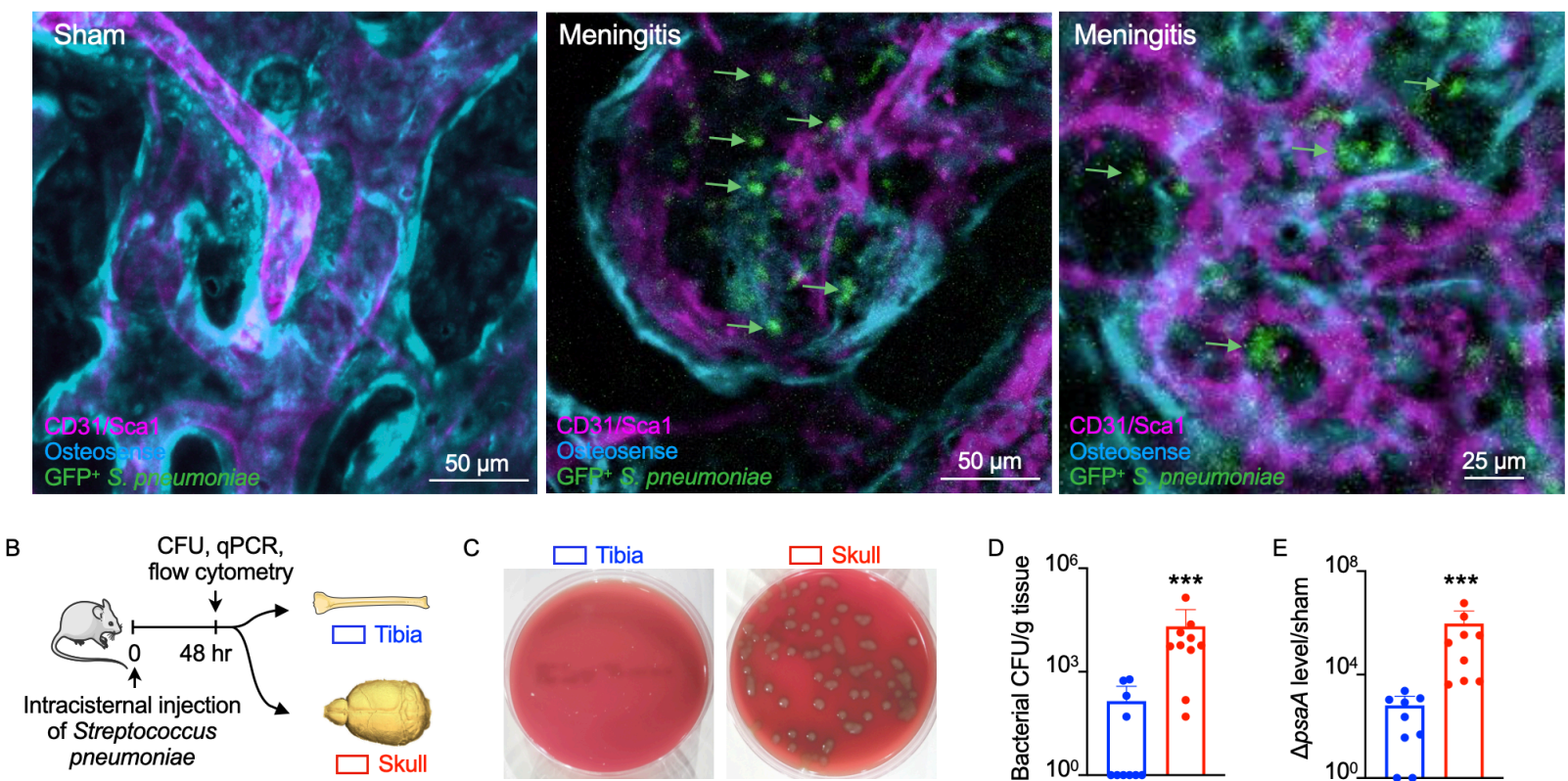

C
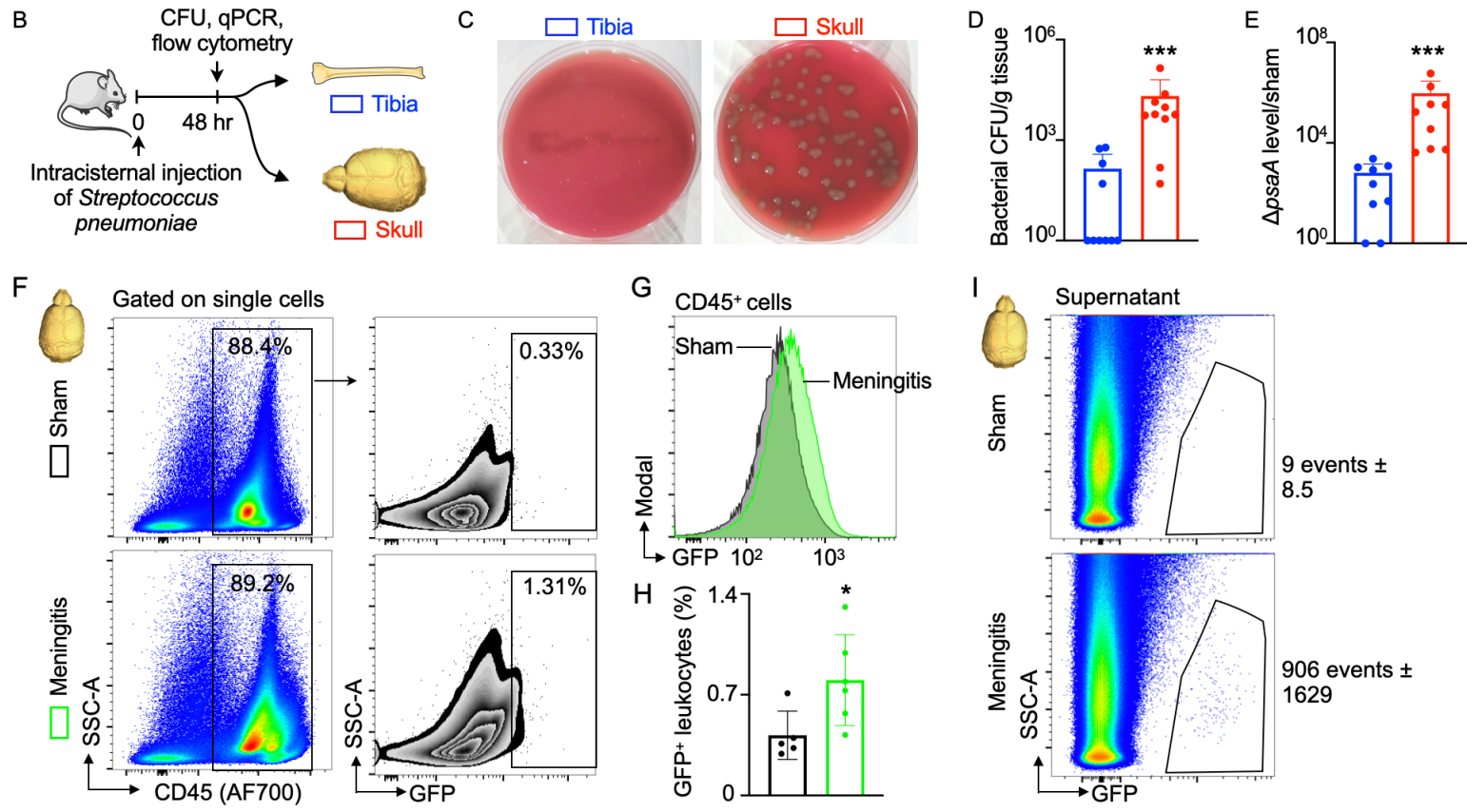

Fig. 3. Bacterial migration to skull marrow. (A) IVM of skull marrow from sham controls injected with artificial CSF (aCSF) or mice $48 \mathrm{hrs}$ after intracisternal injection of GFP+ Streptococcus pneumoniae JWV500 show bacterial presence adjacent to vasculature within the bone marrow ( $\mathrm{n}=3-4$ mice per group; scale: $50 \mu \mathrm{m}$, scale: $25 \mu \mathrm{m}$ ). (B) Experimental outline for experiments in (C-G). (C) Bacterial cultures from mice with pneumococcal meningitis relative to sham controls. (D) Quantitation of bacterial colony forming unit (CFU) assay in (C) shows minimal bacteria in the tibia relative to the skull (mean $\pm S D$; $n=10$ per group; Mann-Whitney test; $\left.{ }^{* *} P<.001\right)$. (E) qPCR of $S$. pneumoniae surface adhesion gene (psaA) expression in tibia versus skull marrow normalized to sham controls (mean $\pm S D ; n=9$ mice; Mann-Whitney test; $\left.{ }^{* * * *} P<.0001\right)$. (F) Gating strategy for CD45+ leukocytes that have interacted with GFP+ $S$. pneumoniae. (G) Histogram of GFP signal in CD45+ leukocytes obtained from mice with meningitis compared to CD45 ${ }^{+}$cells from sham-injected controls. (H) Quantitation of GFP+CD45+ cells analyzed in $(F, G)$ (mean $\pm \mathrm{SD} ; \mathrm{n}=5$ mice; unpaired t-test; $\left.{ }^{*} P<.05\right)(\mathrm{I})$ Extracellular GFP+ $S$. pneumoniae detected by flow cytometry in supernatant of skull marrow from mice with meningitis compared to sham controls ( $n=2-4$ mice per group). 

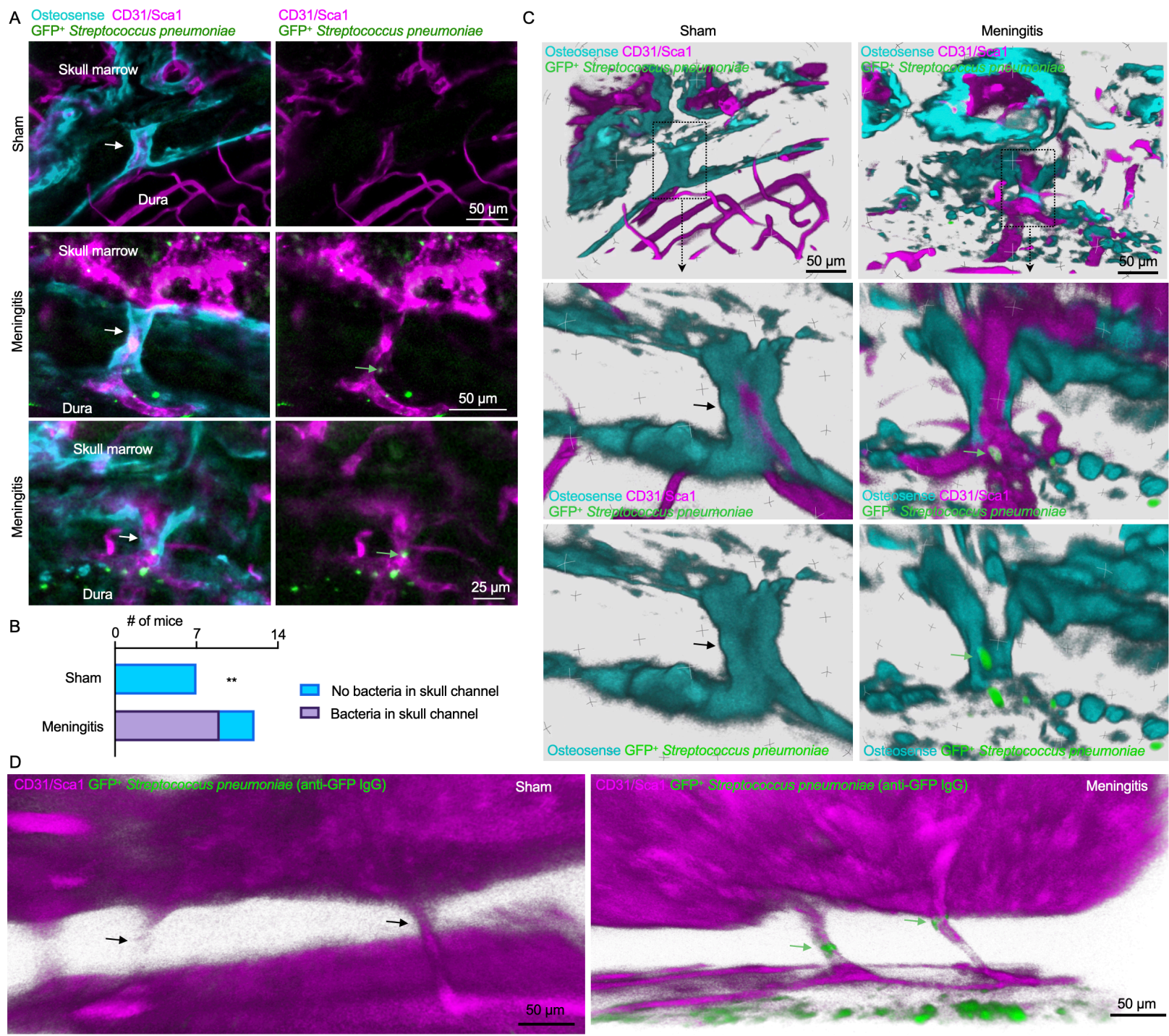

Fig. 4. Skull channels are conduits for pneumococcal migration into the cranial marrow.

(A) Whole-mount ex vivo imaging of skull channels in sham controls or mice after intracisternal injection of GFP+ Streptococcus pneumoniae JWV500 after tissue clearing. Images depict bacteria in skull channels which are visualized using osteosense to label bone and marrow vasculature using a CD31-Sca1 cocktail ( $n=7-11$ mice per group; scale: $50 \mu \mathrm{m}$ or $25 \mu \mathrm{m}$ as indicated). (B) Quantitation of S. pneumoniae GFP signal in skull channels in sham controls and mice with meningitis $\left(n=7\right.$ controls, $n=11$ mice with meningitis; Chi-Square Test; $\left.{ }^{* *} P<.01\right)$. (C) 3D renderings of $(A)$ show intra-channel perivascular localization of $S$. pneumoniae (scale: $50 \mu \mathrm{m}$ ). (D) Whole-mount ex vivo imaging after CUBIC tissue processing for GFP detection after intracisternal injection of GFP+ Streptococcus pneumoniae JWV500. CUBIC protocol (described in methods) was followed by immunostaining for bacterial GFP. Bone marrow vasculature was labeled in vivo with CD31-Sca1 ( $\mathrm{n}=2$ mice; scale: $50 \mu \mathrm{m})$. 


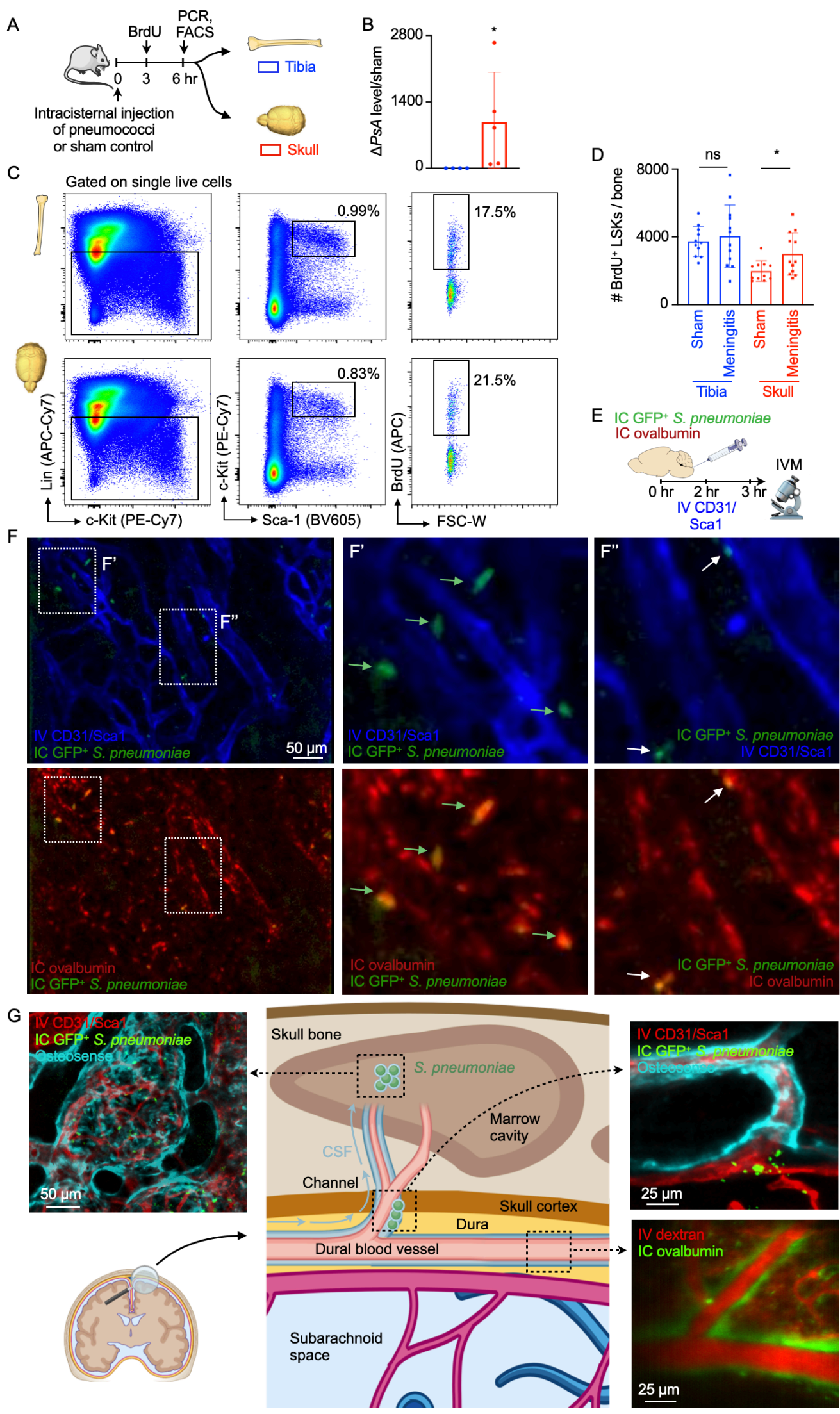

Fig. 5. Bacterial meningitis induces LSK proliferation in the skull. (A) Outline for experiments (B-D). (B) qPCR detection of $S$. pneumoniae psaA gene expression in tibia versus 
skull normalized to sham controls (mean $\pm S D$; $n=4$ mice; Mann-Whitney test; ${ }^{*} P<.05$ ). (C) Flow cytometry gating of tibia and skull bone marrow. (D) Quantitation of BrdU ${ }^{+}$lineage- Sca- $1^{+} \mathrm{C}_{-} \mathrm{kit}^{+}$ hematopoietic progenitors (mean $\pm S D ; n=11-12$ mice per group; unpaired t-test; $n s=$ not significant, $\left.{ }^{*} P<.05\right)$. (E) Experimental outline. (F) IVM of skull BM in mice injected with GFP+ Streptococcus pneumoniae JWV500 to interrogate bacterial propagation 4-6 hours after intracisternal injection. Bone marrow vasculature was visualized after intravenous injection of fluorescently labeled antibodies for CD31 and Sca1 and CSF outflow by intracisternal injection of ovalbumin. F' inset depicts large GFP+ bacterial areas (arrows). F" depicts smaller GFP+ areas, presumably bacterial colonies ( $n=2$ mice). (G) Summary cartoon indicates CSF outflow via dural perivascular spaces through skull channels into the cranial marrow; this route is usurped by bacteria during meningitis. 


\section{Materials and Methods}

\section{Mice}

Mice were housed under certified pathogen-free conditions at Massachusetts General Hospital's Center for Comparative Medicine. All experiments were conducted in accordance with the Institutional Animal Care and Use Committee's approval (Protocols: 2005N000306, 2019N000203, 2009N000085 and 2007N000148). Male and female C57BL/6 (WT; JAX 000664) age 10-16 weeks were used for all experiments. Mice were provided rodent chow and water and allowed to acclimate for 1 week before experimentation. All mice were group-housed with ad libitum access to food and water. Animals were assigned to experimental groups randomly, and experiments were performed in a blinded fashion.

\section{Streptococcus pneumoniae}

Streptococcus pneumoniae strain JWV500 (D39 hlpA-gfp_Camr; serotype 2) was generously provided by Dr. Jan-Willem Veening and prepared as previously described (26). Streptococcus pneumoniae Xen10 (serotype 3) was purchased from Caliper LifeSciences. GFP+ S. pneumoniae and S. pneumoniae Xen10 were cultured in brain heart infusion broth (BHI) containing $4.5 \mu \mathrm{g} / \mathrm{ml}$ chloramphenicol or $200 \mu \mathrm{g} / \mathrm{ml}$ kanamycin, respectively. Streptococcus pneumoniae strains were cryopreserved in $\mathrm{BHI}$ with $30 \%$ glycerol, thawed the day before the experiment in a $37^{\circ} \mathrm{C}$ water bath and diluted in fresh $\mathrm{BHI}$ with the antibiotic. Bacterial doses between $1: 10^{3}$ and 1:106 CFUs were used, depending on experimental design. Optical densities $(600 \mathrm{~nm})$ were used for all bacterial experiments to approximate CFUs, but serial plating dilutions confirmed actual CFUs on $\mathrm{BHI}$ agar.

\section{MicroCT protocol and channel analysis}

Samples were imaged using a high-resolution CT scanner ( $\mu$ CT40, Scanco Medical AG, Brüttisellen, Switzerland). Data were acquired using a $6 \mu \mathrm{m}^{3}$ isotropic voxel size, $70 \mathrm{kVp}$ peak X-ray tube intensity, $114 \mu \mathrm{A} X$-ray tube current and $200 \mathrm{~ms}$ integration time, and they were subjected to Gaussian filtration. Image renderings were done in Amira (FEI, Hillsboro, OR). Prior 
to skull channel quantification in Volocity, DICOM files were converted into ND2 file format. Three regions of interest in the midline from the right frontal, parietal and occipital bones were identified and analyzed. Skull channels within regions of interest were visualized on an angled coronal view. Individual channels were given unique identifiers followed by length and width measurements for each channel.

\section{Cisterna magna injections}

Mice were anesthetized by isoflurane inhalation (3-4\% induction, 1-2\% maintenance), injected with buprenorphine $(0.1 \mathrm{mg} / \mathrm{kg}$ before surgery and every $12 \mathrm{hrs}$ until sacrifice), followed by hair removal from the back of the neck (Nair). We used a thermometer and feedback-controlled heating blanket (Harvard Apparatus) to maintain body temperature at $37^{\circ} \mathrm{C}$. Mice were fixed on a stereotaxic frame (Harvard Apparatus) with the neck angled downward for optimal cisterna magna exposure, and eye ointment (Dechra) was applied to prevent indirect damage during surgery. An approximately $4 \mathrm{~mm}$ vertical skin incision was made at the back of the neck, and the junction between the skull and the $1^{\text {st }}$ vertebrae was exposed by bluntly separating the overlaying muscles (10). A $30 \mu \mathrm{m}$ inner diameter glass micropipette (Fivephoton Biochemicals MGM-1C-30-30) attached to a ultra-precise micro manipulator (Stoelting) loaded with $5 \mu$ of artificial CSF (119 mM NaCl, $26.2 \mathrm{mM} \mathrm{NaHCO}_{3}, 2.5 \mathrm{mM} \mathrm{KCl}, 1 \mathrm{mM} \mathrm{NaH} \mathrm{PO}_{4}, 1.3 \mathrm{mM} \mathrm{MgCl}$, $2.5 \mathrm{mM} \mathrm{CaCl}_{2}$ ) containing $5 \times 10^{3}$ or $1 \times 10^{5}$ Streptococcus pneumoniae was inserted through the dura towards the cerebellopontine angle. For sham controls, $5 \mu$ of sterile artificial CSF was injected. These injections were administered at a speed of $1 \mu \mathrm{l} / \mathrm{min}$ with a micro syringe pump (Harvard Apparatus) and a custom-order Hamilton Company syringe (10 $\mu$ l volume; 3-point style; 20 gauge; $10 \mathrm{~mm}$ needle length). In order to prevent backflow-induced variability among individual injections, the needle was retracted incrementally over the course of 10-30 minutes. After the injection, the incision and surrounding area were treated with Terramycin ointment (Zoetis). The incision was sutured with a 5-0 silk suture (Ethilon). 
Surgical preparation for 2-photon IVM (described below) was similarly performed with slight modifications to minimize pain and distress over longer imaging periods. Mice were anesthetized with $3 \%$ isoflurane, placed on a stereotactic surgery frame (Harvard Apparatus) and then maintained on $1.5 \%$ isoflurane in $100 \%$ oxygen. Mice were also treated with $0.05 \mathrm{mg}$ per $100 \mathrm{~g}$ body weight of glycopyrrolate (Baxter, Inc.), $0.025 \mathrm{mg}$ per $100 \mathrm{~g}$ body weight of dexamethasone (07-808-8194, Phoenix Pharm, Inc.) and $0.5 \mathrm{mg}$ per $100 \mathrm{~g}$ of ketoprofen (Zoetis, Inc.). Glycopyrrolate and ketoprofen were injected intramuscularly, while dexamethasone was injected subcutaneously. Bupivacaine $(0.1 \mathrm{ml}, 0.125 \%$; Hospira, Inc.) was subcutaneously administered at the incision site to provide a local nerve block. Animals were provided with $1 \mathrm{ml}$ per $100 \mathrm{~g}$ of $5 \%(\mathrm{w} / \mathrm{v})$ glucose in normal saline subcutaneously every hour during the procedure. We used a thermometer and feedback-controlled heating blanket (40-90-8D DC, FHC) to maintain body temperature at $37^{\circ} \mathrm{C}$. The head and neck were shaved and washed with $70 \%$ (v/v) ethanol and iodine solution (AgriLabs). An intracisternal injection was performed as described above. After the injections, the exposed tissue was sealed with cyanoacrylate adhesive (Loctite) and dental cement (Co-Oral-Ite Dental).

\section{Skull marrow preparation and confocal microscopy}

For in vivo imaging, the mouse head was shaved and held in a stereotactic skull holder (Harvard Apparatus). Calvarial bone marrow was exposed, as previously described (11), by incising a skin flap and then applying glycerol (Sigma-Aldrich) to prevent tissue drying. Skull marrow microscopy was performed with a single photon confocal microscope (IV 100, Olympus, Tokyo, Japan) equipped with IV10-ASW 01.01.00.05 software (Olympus). A field of view at 4x magnification covers a $2290 \mu \mathrm{m}$ x $2290 \mu \mathrm{m}$ area while 20x magnification covers a $458 \mu \mathrm{m}$ × 458 $\mu \mathrm{m}$ area consisting of $512 \times 512$ pixels. For ex vivo skull preparation and imaging after tissue clearing, mice were humanely sacrificed. Then, the head with brain intact was split longitudinally along the sagittal sinus and fixed for 1 hour in $4 \%$ paraformaldehyde, after which tissue was washed and subjected to clearing in RapiClear 1.49 (SunJinLab) for 1-2 hours, depending on sample size. Specimen were then mounted on a custom-built tissue holder with a hanging, 
mounted coverslip (Harvard Apparatus). As indicated in specific figure panels with regard to the intravenous injection timing (retro-orbital, $100 \mu$ total volume in PBS), mice received the following reagents for IVM and ex vivo imaging: $30 \mu$ I CD31-AF647 (Biolegend, MEC13.3), $30 \mu l$ CD31-PE (Biolegend, clone MEC13.3), $30 \mu$ Sca1-AF647 (Biolegend, clone D7), 30 Il Sca1-PE (Biolegend, clone D7) and $100 \mu \mathrm{l}$ Osteosense 750EX (Perkin Elmer Cat\#NEV10053EX).

\section{2-Photon IVM and ex vivo imaging}

Mice were anesthetized and placed on a custom stereotactic frame. During imaging, anesthesia was maintained with $\sim 1.5 \%$ isoflurane in $100 \%$ oxygen, with small adjustments to maintain the respiratory rate at $\sim 1 \mathrm{~Hz}$. To fluorescently label the microvasculature, Texas red dextran (40 $\mu$, $2.5 \%$, molecular weight $(\mathrm{MW})=70,000 \mathrm{kDA}$, Thermo Fisher Scientific) in saline was injected retro-orbitally immediately before imaging. Three-dimensional data sets of the calvarial bone marrow, meninges, meningeal vasculature and CSF transport were obtained using a custombuilt two-photon excitation microscope. Imaging was done using $830 \mathrm{~nm}, 120 \mathrm{fs}$ pulses from a Ti:Sapphire laser oscillator (Spectra-Physics InSight X3). The laser beam was scanned by polygon scanners (30 frames s-1) and focused into the sample using a $60 x$ water-immersion objective lens for high-resolution imaging (numerical aperture of 1.1, Olympus). The emitted fluorescence was detected on photomultiplier tubes through the following emission filters: 400/60 nm for second harmonic generation (SHG), 525/50 nm for Alexa488/FITC and 605/50 $\mathrm{nm}$ for Texas red. Laser scanning and data acquisition were controlled by custom-built software. Stacks of images were spaced at $1 \mu \mathrm{m}$ axially.

\section{Cerebrospinal fluid tracing}

Mice were injected with fluorescent CSF tracers which were reconstituted in artificial cerebrospinal fluid (aCSF) at a concentration of $0.5 \%$. For 2-photon IVM and 2-photon ex vivo experiments, mice were IC injected with 2000kD FITC-Dextran (Thermo Fisher Cat\# D7137), 70kD Texas Red-Dextran (Thermo Fisher Cat\# D1830) and AF647-Ovalbumin (Invitrogen Cat\# O34784). For confocal microscopy, mice were injected with AF488-Ovalbumin (Sigma Cat\# 
O34781) and AF647-Ovalbumin (Sigma Cat\# O34784) reconstituted in a $5 \mu$ l volume of aCSF. We examined CSF trafficking in the marrow by ex vivo imaging as described recently(4). Zstacks of marrow-channnel-dural spaces were acquired in vivo, after which mice were euthanized at the indicated time points following intracisternal injection of tracer. A piece of frontal bone containing marrow was then excised, preserving the integrity of the dura and the bone marrow cavities. The excised specimen was inverted and rapidly transferred into an aCSF bath or stage-mounted for whole-mount imaging. Tibia bone was embedded in OCT medium (TissueTek) and snap-frozen. Cortical bone was shaved on a crystotat until the marrow was visible.

\section{Transmission electron microscopy}

Skull samples were collected for TEM from naive mice as described above with the following modifications. Cardiac perfusion with PBS was followed by $20 \mathrm{ml}$ of Karnovsky's fixative $(0.1 \mathrm{M}$ sodium cacodylate, $2.5 \%$ glutaraldehyde, $2 \%$ paraformaldehyde). The skull was excised and trimmed to contain the intact frontal, parietal and occipital bones and fixed for $3 \mathrm{hrs}$ in Karnovsky's fixative followed by $48 \mathrm{hr}$ fixation at $4^{\circ} \mathrm{C}$. Skulls were then trimmed to marrowcontaining $2 \times 4$ mm pieces and decalcified over 2 weeks in 140 mM EDTA (pH 7.4; Boston BioProducts). Decalcification solution was replaced with fresh solution every other day and on the final day samples were washed in $0.1 \mathrm{M}$ sodium cacodylate buffer for TEM preparation.

After several washes in PBS, followed by several rinses in cacodylate buffer, specimens were fixed in $1 \%$ glutaraldehyde in cacodylate buffer overnight at $4^{\circ} \mathrm{C}$. The following day, specimens were rinsed several times with cacodylate buffer, infiltrated $1 \mathrm{hr}$ in $1 \%$ osmium tetroxide, rinsed several times again in cacodylate buffer and then dehydrated through a graded series of ethanols to $100 \%$. Samples were dehydrated briefly in $100 \%$ propylene oxide, then incubated in a 1:1 mix of propylene oxide and Eponate resin (Ted Pella, Redding, CA) overnight at room temperature on a gentle rotator. The following day, specimens were incubated at least $3 \mathrm{hr}$ in $100 \%$ Eponate resin, then placed into flat molds with fresh $100 \%$ Eponate resin and allowed to 
polymerize in a $60^{\circ} \mathrm{C}$ oven $(24-48 \mathrm{hrs})$. Semi-thin sections $(1 \mu \mathrm{m})$ were collected onto slides and stained with $0.1 \%$ toluidine blue (in $0.1 \%$ sodium borate) to preview and confirm the presence of channels. Thin (70nm) sections were cut using a Leica EM UC7 ultramicrotome, collected onto formvar-coated grids, stained with $2 \%$ uranyl acetate and Reynold's lead citrate and examined in a JEOL JEM 1011 transmission electron microscope at $80 \mathrm{kV}$. Images were collected using an AMT digital imaging system with proprietary image capture software (Advanced Microscopy Techniques, Danvers, MA).

\section{Clear unobstructed brain/body imaging cocktail and computational analysis (CUBIC)}

This protocol was adapted from Tainaka and colleagues (15) and used to detect GFP expressed by $S$. pneumoniae in skull channels. Mice were first intracisternally injected with GFP+ $S$. pneumoniae as described above for analysis 48 hours after infection. One hour prior to sacrifice, mice were retro-orbitally injected with CD31/Sca1-AF647 cocktail to label the vasculature. Mice were perfused with PBS followed by 4\% PFA prior to skull removal. Skull bones were fixed overnight in $4 \%$ PFA with shaking at $4^{\circ} \mathrm{C}$ and subsequently washed $5 x$ for 10 minutes/wash in PBS on a bench-top shaker (400 RPM). Skull samples were delipidized/ decolored in CUBIC-L solution (10 wt\% N-butyldiethanolamine (Tokyo Chemical Industry CU\#0414), 10 wt\% Triton X-100(Sigma)) for 4 days while rotating at $37^{\circ} \mathrm{C}$. CUBIC-L solution was refreshed on day 3 , and on the final day samples were washed $5 x$ with PBS. Skulls were decalcified over the course of 5 days in CUBIC-B solution (10 wt\% EDTA (Boston BioProducts), 15 wt\% imidazole (Tokyo Chemical Industry CU\#1352)) at $37^{\circ} \mathrm{C}$ while rotating. Solution was refreshed on day 3 , and on the final day samples were washed with PBS. Skull samples were then immersed in CUBIC-L for 2 days at $37^{\circ} \mathrm{C}$ while rotating and then washed with PBS prior to immunostaining steps. Primary antibody staining using chicken anti-GFP (Abcam, ab13790, 1:300) was performed in staining buffer comprised of PBS with $1 \%$ triton-x (Sigma), 10\% normal goat serum (Vector Labs) and $0.2 \%$ sodium azide (Sigma) for 4 days while gently shaking (200 RPM) at room temperature. Samples were then washed with CUBIC wash buffer (PBS with 1\% triton-x) at room temperature $3 x$ for 1 hour per wash after which samples were incubated in goat 
anti-chicken AF555 (ThermoFisher, A-21437) secondary antibody. This staining was performed in the same buffer used for the primary antibody incubation step at room temperature for 3 days. Samples were washed in PBS 3x for 1 hour per wash and subjected to refractive index matching in CUBIC-R (45 wt\% antipyrine (Tokyo Chemical Industry CU\#0640), 30 wt\% nicotinamide (Tokyo Chemical Industry CU\#0855) for 2 days at room temperature, after which samples were ready for whole-mount confocal imaging.

\section{Bioluminescence imaging}

Studies utilizing $S$. pneumoniae Xen10 followed the intracisternal injection technique described above. Briefly, $5 \times 10^{3}$ S. pneumoniae Xen10 or an equivalent volume of aCSF was injected into cohorts of age-matched mice for imaging at 12, 36 and 48 hours following injection to track the development of meningitis. Images were acquired with an AmiX BLI/X-Ray Scanner (AmiX) using medium binning and a 3 min exposure time across all time points. Photon intensity was scaled at a range of $4 \times 10^{4}-2 \times 10^{7}$ photons $/ \mathrm{cm}^{2}$ to allow for cross-group comparisons. The signal was quantified with AMIView software by defining a region of interest across the head, neck and spine. This region of interest was then uniformly fitted to each individual mouse.

\section{Bacterial colony forming unit assay}

CFU assays from blood and CSF of sham and meningitis mice were performed 48 hours after injection. Mice were fixed onto a stereotactic frame in a manner similar to the orientation used for intracisternal injections. To sample the CSF, the injection site was reopened and the dura mater punctured with a glass micropipette to aspirate 5-10 $\mu$ l CSF. Mice were then removed from the frame and approximately $100 \mu \mathrm{l}$ of blood was collected by cardiac puncture using a 23 gauge needle and syringe pre-rinsed with 2 mM EDTA. For skull and tibia CFUs, bones were aseptically harvested from sham and meningitis groups, and the meninges were dissected from the skull in sterile PBS containing 5\% BSA/2 mM EDTA. Dissection was performed under a stereotactic microscope using fine forceps (\#5, angled) and angled spring scissors (Fine Science Tools). Pilot experiments were performed to titer dilutions of CSF, blood, skull or tibia 
homogenate necessary to visualize bacterial growth on blood agar plates containing $50 \mu \mathrm{g} / \mathrm{ml}$ kanamycin (TEKnova Cat\#T0194). A cell-spreader was used to evenly distribute homogenates across the plate and the plate was stored in a $37^{\circ} \mathrm{C}$ for 24 hours prior to analysis. Plates were photographed and colonies quantitated for relative comparisons.

\section{S. pneumoniae detection by $q P C R$}

We analyzed relative amounts of the $S$. pneumoniae gene psaA from tibias and skulls excised from sham and meningitis cohorts of adult mice 48 hours after intracisternal injection of aCSF or S. pneumoniae Xen10. Injections were performed as described above and bones were aseptically excised from mice. All mice were perfused with $20 \mathrm{~mL}$ PBS prior to removal of tibia and skull bones. After bones were excised, the meninges were dissected from the skull bone, after which tibia and skull bones were snap-frozen in liquid nitrogen and stored at $-80^{\circ} \mathrm{C}$ overnight prior to subsequent analysis. DNA isolation protocol and primers used to detect $S$. pneumoniae psaA were adapted from an established protocol (27). Briefly, 3 primers were custom synthesized (IDT) psaA forward (5'-GCCCTAATAAATTGGAGGATCTAATGA-3'), psaA reverse (5'-GACCAGAAGTTGTATCTTTTTTTCCG-3') and psaA probe (5'-HEXCTAGCACATGCTACAAGAATGATTGCAGAAA GAAA-3'-phosphate) for qPCR-based relative expression analysis. Skull and tibia bones were trimmed to $50 \mathrm{mg}$ and homogenized with fine scissors in $180 \mu \mathrm{l}$ ALT buffer containing $0.04 \mathrm{~g} / \mathrm{ml}$ lysozyme (Sigma) and $75 \mathrm{U} / \mathrm{ml}$ of mutanolysin (Sigma). Digestion was performed for 1 hour at $37^{\circ} \mathrm{C}$ in a shaking water bath. Subsequent steps for DNA isolation and purification were performed following manufacturer guidelines from the Qiagen DNA Mini-Kit manual (Qiagen). gDNA for each sample was spectrophotometrically measured (ThermoFisher NanoDrop 2000) and equivalent DNA amounts were loaded for subsequent qPCR. As described in Carvalho et al., reactions were allowed to run for 45 thermal cycles for amplification and in duplicate for all samples analyzed. Cycle threshold values above 40 were considered negative.

\section{Flow cytometry}


To assess skull and tibia bone marrow hematopoietic cells, mice were anesthetized, sacrificed and perfused with $20 \mathrm{~mL}$ PBS to remove blood cells. Tibia and skull were excised, and then meninges were removed from the skull and mechanically homogenized in homogenization buffer (PBS with 5\% BSA and 2 mM EDTA). Homogenate was filtered through a $40 \mu \mathrm{m}$ strainer, centrifuged for 5 ' at $340 \mathrm{~g}$ and resuspended in FACS buffer (PBS with 0.5\% BSA) for surface antibody staining. To analyze hematopoietic stem and progenitor cells, cells were first stained with biotin-conjugated anti-mouse antibodies against CD3 (BioLegend, clone 145-2C11), CD4 (BioLegend, clone GK1.5), CD8a (BioLegend, clone 53-6.7), CD49b (BioLegend, clone DX5), CD90.2 (BioLegend, clone 30-H12), CD19 (BioLegend, clone 6D5), B220 (BioLegend, clone RA3-6B2), NK1.1 (BioLegend, clone PK136), TER119 (BioLegend, clone TER-119), CD11b (BioLegend, clone M1/70), CD11c (BioLegend, clone N418) and Gr1 (BioLegend, clone RB6-8C5 all diluted 1:300), which all served as lineage (Lin) markers, and LIVE/DEAD Fixable Aqua Dead Cell Stain (Life Technologies, 1:300). Staining was done for 30 minutes on ice followed by a wash/spin and resuspension in FACs buffer for secondary staining. LSK analysis was performed by staining cells with ckit-PE-Cy7 (BioLegend, clone 2B8), Sca1-BV605 (BioLegend, clone D7) and Streptavidin-APC-Cy7 (BioLegend, 1:100). LSK were identified as Lin- c-kit+ Sca-1+. Antibodies listed in the secondary staining panel were used at 1:100 dilution in a $500 \mu \mathrm{l}$ single-cell suspension volume for 30 minutes on ice. Cells were further stained with the APC BrdU Flow Kit (552598, BD Biosciences) following the manufacturer's guidelines for analysis. BrdU was administered via intraperitoneal injection 3 hours before sacrifice to analyze LSK proliferation 6 hours after intracisternal bacteria injection. Cells were then washed with FACS buffer, spun down at $340 \mathrm{~g}$ for 5 minutes and resuspended in $400 \mu$ l of FACS buffer for analysis. Events were recorded on an LSRII flow cytometer and accompanying FACS DIVA 6.1 software (BD Biosciences). Data were analyzed with FlowJo 10 software (Becton Dickinson).

\section{Flow cytometric detection of bacteria}

Mice were intracisternally injected with GFP+ S. pneumoniae as described above and analyzed 48 hours after injection. Skulls were aseptically excised followed by meningeal dissection under 
a light microscope, as previously described. Samples were then mechanically homogenized in homogenization buffer (PBS with 5\% BSA and $2 \mathrm{mM}$ EDTA), filtered through a $40 \mu \mathrm{m}$ strainer and spun down for $5 \mathrm{~min}$ at $340 \mathrm{~g}$. Supernatant containing bacteria was isolated and further spun at $5000 \mathrm{~g}$ for $10 \mathrm{~min}$ and resuspended in $400 \mu \mathrm{l}$ to analyze free-floating bacteria. Cell pellets were stained with CD45-AF700 (Biolegend, clone 30F11, 1:300) in FACS buffer on ice for 30 minutes. Samples were washed with $2 \mathrm{ml}$ FACS buffer, spun down at $340 \mathrm{~g}$ for $5 \mathrm{~min}$ and resuspended in FACS buffer for analysis. Events were recorded on an LSRII flow cytometer and accompanying FACS DIVA 6.1 software (BD Biosciences). Data were analyzed with FlowJo 10 software (Becton Dickinson). Cells were gated for single cells by FSC and SSC parameters, as previously described, and leukocytes were defined as CD45+ cells. GFP+CD45+ cells were discerned for GFP-CD45+ cells, and mean fluorescence intensity was quantitated across groups.

\section{RNA extraction and $q P C R$}

RNA was isolated from meninges using the RNeasy Micro kit (Qiagen). High-Capacity RNA to cDNA kit (Applied Biosystems) was used for first-strand cDNA synthesis from meningeal RNA. TaqMan gene expression kits were utilized to measure target genes of interest: $/ /-1 \beta$ (Mm00434228_m1, ThermoFisher), II-6 (Mm00446190_m1, ThermoFisher) and TNF- $\alpha$ (Mm00443258_m1, ThermoFisher). Target gene primers were all FAM-MGB and all target gene relative expression analyses were normalized to a housekeeping gene, Gapdh (VIC-MGB, Mm99999915_g1, ThermoFisher). Samples were run on a 7500 Real-Time PCR machine (Applied Biosystems).

\section{Image analysis}

Images were processed and analyzed using FIJI version 2.1.0, MATLAB R2015b (Mathworks) or Volocity 3D imaging software version 6.3 (PerkinElmer). To complete ex vivo 2-PM channel 
analysis for CSF tracer presence, z-stacks were untilted using a MATLAB code developed inhouse, such that the skull surface spanned the least number of $z$ depths, i.e. it lay approximately flat. Afterwards, enhanced contrast was performed on each channel $(R, G, B)$ to correct for intensity attenuation due to light absorption in the tissue. The MATLAB version used was MATLAB 2020b. For confocal microscopy images, z-stacks of .75, 1.0 or $2.0 \mu \mathrm{m} /$ slice were taken depending on the imaging objective $(4 \mathrm{x}, 10 \mathrm{x}$ or $20 \mathrm{x})$. These conditions were consistent across all single-photon confocal microscopy images in both ex vivo and intravital microscopy experiments. Images were 3D max-intensity or sum-intensity projected using FIJI and background subtracted followed by an automated de-speckling protocol to remove specklebased noise. Manual thresholding and contrast adjustment were applied uniformly across all samples when necessary. To ensure processing step uniformity, FIJI macros were recorded for the first image processed and then applied to all images from the same cohort. Where indicated, certain images were further analyzed using Volocity software for preparing 3D surface reconstructions. 3D rendering was automatically generated and image contrast, density and brightness manually and uniformly set across all images analyzed. Movies were manually generated in Volocity using snapshots of post-processed datasets and then incorporated into a single movie in AVI format.

\section{Statistics}

All statistical analyses were performed using GraphPad Prism (GraphPad Software v9.2). All quantitative results are reported as the mean \pm standard deviation. For single variable comparisons of parametric datasets for two groups, an unpaired t-test was performed. For nonparametric datasets of unpaired data, a Mann-Whitney test was performed. For parametric datasets for multiple groups, a one-way ANOVA was performed with a post-hoc correction for multiple comparisons as indicated in individual legends. For non-parametric datasets for multiple groups, a Kruskal-Wallis test was performed followed by post-hoc correction for multiple comparisons where indicated. 

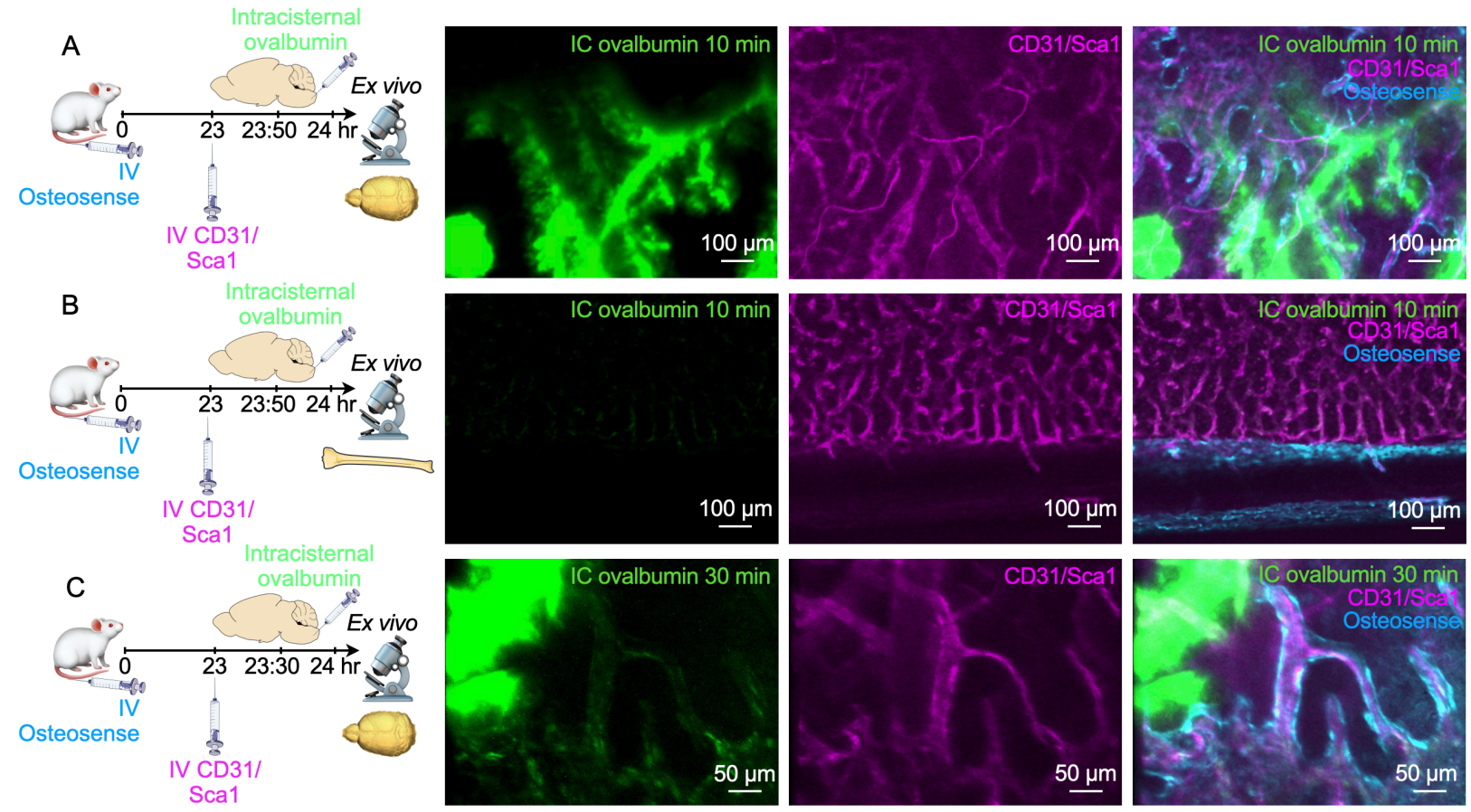

Fig. S1. Dynamics of CSF outflow into bone marrow. (A) Ex vivo imaging of whole-mount skull 10 min after intracisternal (IC) injection of ovalbumin. Intravenous(IV) injection of CD31/ Sca1 labeled vasculature and IV osteosense the bone. (B) Ex vivo imaging of tibia 10 minutes after intracisternal injection of ovalbumin. (C) Imaging 30 minutes after intracisternal injection of ovalbumin. 


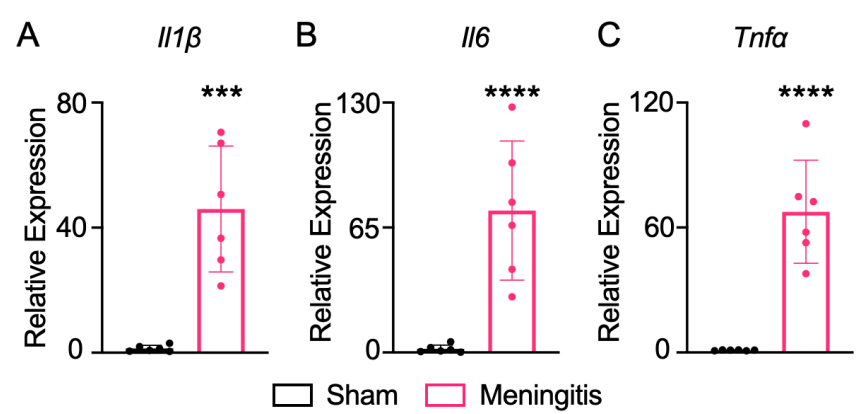

Fig. S2. Inflammation in the meninges. qPCR analysis of meninges isolated from either sham controls that were intracisternally injected with artificial CSF or mice 48 hours after intracisternal infection for relative expression analysis of $(\mathbf{A}) / / 1 \beta,(\mathbf{B}) / / 6$ and (C) Tnfo (mean \pm SD; $n=6$ mice per group; unpaired t-test; ${ }^{* * *} P<.001$ ). 


\section{Movie S1.}

Intravital microscopy of perivascular CSF tracer flow along a dural vessel. Intravenously injected dextran is shown in red, intracisternally injected ovalbumin in green.

\section{Movie S2.}

Ex vivo acquired z-stack of the internal skull cortex showing the CSF tracer (intracisternally injected dextran, green) traveling along channel vessels (intravenously injected dextran, red) into the marrow.

\section{Movie S3.}

Three-dimensional rendering of an optically cleared skull-brain specimen highlights channels in a sham control mouse. Vasculature was labeled with intravenously injected CD31/Sca-1 antibodies and bone with osteosense.

\section{Movie S4.}

Three-dimensional rendering of an optically cleared skull-brain specimen from a mouse with meningitis 48 hours after intracisternal injection of GFP+ $S$. pneumoniae shows bacterial propagation (green) in the subarachnoid space adjacent to skull channels.

\section{Movie S5.}

Three-dimensional rendering of a maximal intensity projection acquired by intravital microscopy of skull marrow in a sham control mouse.

\section{Movie S6.}

Three-dimensional rendering of a maximal intensity projection acquired by intravital microscopy of skull marrow containing GFP+ S. pneumoniae (green) in a mouse with meningitis.

\section{Movie S7.}

Three-dimensional rendering of skull channels in a sham control mouse. Vasculature was labeled with intravenous injection of CD31/Sca-1 antibodies and bone with osteosense.

\section{Movie S8.}

Three-dimensional rendering of a skull channel in a mouse with meningitis shows $\mathrm{GFP}^{+} \mathrm{S}$. pneumoniae (green) inside the channel. Vasculature was labeled with intravenous injection of CD31/Sca-1 antibodies and bone with osteosense. 